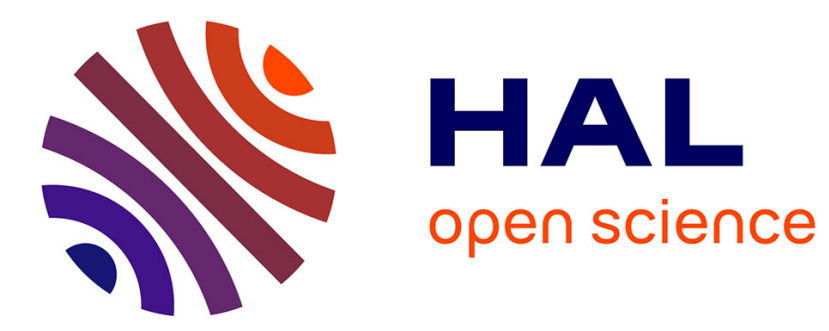

\title{
Contraintes et choix organisationnels dans les dispositifs de monnaies sociales
}

Jérôme Blanc

\section{To cite this version:}

Jérôme Blanc. Contraintes et choix organisationnels dans les dispositifs de monnaies sociales. Annals of Public and Cooperative Economics, 2009, 80 (4), pp.547-577. 10.1111/j.1467-8292.2009.00398.x . halshs-00709199

\section{HAL Id: halshs-00709199 \\ https://shs.hal.science/halshs-00709199}

Submitted on 18 Jun 2012

HAL is a multi-disciplinary open access archive for the deposit and dissemination of scientific research documents, whether they are published or not. The documents may come from teaching and research institutions in France or abroad, or from public or private research centers.
L'archive ouverte pluridisciplinaire HAL, est destinée au dépôt et à la diffusion de documents scientifiques de niveau recherche, publiés ou non, émanant des établissements d'enseignement et de recherche français ou étrangers, des laboratoires publics ou privés. 


\section{CONTRAINTES ET CHOIX ORGANISATIONNELS DANS LES DISPOSITIFS DE MONNAIES SOCIALES}

Published in the journal Annals of Public and Cooperative Economics / Annales de l'économie publique, sociale et coopérative (CIRIEC, International Centre of Research and Information on the Public and Cooperative Economy et Blackwell Publishing)

Article full reference :

Blanc, J. (2009), CONTRAINTES ET CHOIX ORGANISATIONNELS DANS LES DISPOSITIFS DE MONNAIES SOCIALES. Annals of Public and Cooperative Economics, 80: 547-577. doi: 10.1111/j.1467-8292.2009.00398.x

The present version is the author's version.

\section{Author}

Jérôme Blanc

Maître de conférences en sciences économiques à l'Université Lumière Lyon 2

Chercheur au LEFI (Laboratoire d'économie de la firme et des institutions, EA 4012)

Courriel : jerome.blanc@ish-lyon.cnrs.fr

\section{Abstract \\ (Organisational choices and constraints in community or complementary currency schemes)}

This text discusses the organisational choices related to community or complementary currency schemes (like LETS, Argentinean trueque, Time banks, etc) by presenting a set of possibilities and their implications. The key question is that of the viability of the schemes, based on the idea that different objectives require suitable organisational choices. Two main domains are explored: first, the schemes' size and the categories of people and exchanges to be included in; second, the conditions of the issuance, the circulation and the convertibility of the currencies. Each possible option is associated with a set of constraints. The combinations of these constraints result in identifying five models of community or complementary currencies, which seem coherent compared to their objectives.

\section{Résumé}

Ce texte interroge les choix organisationnels relatifs aux dispositifs de monnaies sociales (comme les LETS, Time banks, clubs de trueque argentins, etc.) en présentant un ensemble de possibilités et leurs implications. La question centrale est celle de la viabilité des dispositifs, avec l'idée selon laquelle des objectifs différents requièrent des choix organisationnels appropriés. Deux grands domaines sont explorés : d'abord, la taille des dispositifs et les catégories de personnes et d'échange; ensuite, les conditions de l'émission, de la circulation et de la convertibilité des monnaies. De chacune des options possibles découle un ensemble de contraintes. Les combinaisons de ces contraintes conduisent à identifier cinq modèles de monnaies sociales qui semblent cohérents relativement à leurs objectifs. 


\title{
CONTRAINTES ET CHOIX ORGANISATIONNELS DANS LES DISPOSITIFS DE MONNAIES SOCIALES
}

\begin{abstract}
1. Introduction
Les monnaies sociales sont des dispositifs locaux, qualifiés souvent de «monnaies complémentaires » et parfois de «monnaies communautaires» («complementary currencies» et «community currency systems »), qui fournissent le cadre nécessaire au développement d'échanges de services et de biens entre adhérents d'une association ad hoc et qu'une monnaie interne permet de comptabiliser et de régler. Les multiples présentations de ces dispositifs montrent clairement que, au-delà de mobiles communs, la diversité prévaut et, s'il existe des grands dispositifs connus et diffusés dans plusieurs pays, ils ne sont pas organisés de la même façon et ne visent pas les mêmes objectifs : par exemple, les SEL français (Servet dir., 1999; Laacher, 2003), les LETS britanniques (Williams et alii, 2001), les réseaux dits de troc («trueque ») argentins (Hintze dir., 2003 ; Luzzi, 2005 ; Plasencia et Orzi, dir., 2007), l'Ithaca hour, de la ville d'Ithaca (Etat de New York), aux Etats-Unis (Glover, 1995), etc. Les auteurs qui présentent une vue comparée de ces dispositifs sont conduits à montrer leur diversité et tenter d'en dresser des types (Blanc, 2000 ; Lietaer, 2001 ; Greco, 2001 ; Blanc dir., 2006).

L'histoire de la vague contemporaine de monnaies sociales, celle qui commence dans les années 1980 avec le premier LETS de l'histoire à Comox Valley, sur l'île de Vancouver, au Canada, est celle d'une diffusion de l'idée générale et de quelques modèles mais c'est aussi, et de plus en plus, l'histoire d'expérimentations qui vont dans des directions nouvelles. Ces expérimentations prétendent orienter la monnaie au service d'objectifs pertinents touchant à la dynamique d'espaces locaux (territoriaux ou communautaires). L'histoire de ces dispositifs jusqu'ici donne des leçons ambiguës : le fait même que ce mouvement n'a cessé de prendre de l'ampleur en se recomposant et se diversifiant, ainsi que l'explosion du nombre de dispositifs jusqu'au début des années 2000, semblent valider cette prétention à jouer de la plasticité de la monnaie, mais la taille limitée de chacun des dispositifs et leur faible impact socioéconomique, que tous reconnaissent (sauf dans le cas spectaculaire mais éphémère de l'Argentine en 200102), concourt à un bilan nuancé. Depuis le début des années 2000, un tournant semble s'être opéré et une
\end{abstract}


seconde génération de dispositifs émerge peu à peu. Cette génération n'est plus le résultat d'une dynamique, souvent peu professionnalisée, de la société civile, prenant le caractère convivial d'un tâtonnement pour établir des règles de fonctionnement pertinentes. Au contraire, elle peut être ancrée dans des organisations pérennes comme des organisations non gouvernementales (en particulier, l'ONG néerlandaise Strohalm en Amérique latine), liée à des banques coopératives locales (comme à Fortaleza, au Brésil, ou dans certains länder allemands comme le dispositif du Chiemgauer) ou encore résulter de partenariats institutionnels impressionnants comme le projet SOL en France ${ }^{1}$. Pour ces dispositifs professionnels de la seconde génération, il ne s'agit pas de se lancer un peu au hasard, sur la base d'un mouvement enthousiaste mais désordonné, et de partir du principe que «viendra qui voudra » : il s'agit d'imaginer les solutions les plus pertinentes dans le contexte local, selon les besoins locaux, et en cherchant à optimiser leur impact local.

Dans ce contexte, le but de ce texte est de mettre en lumière l'étendue des choix organisationnels possibles pour des dispositifs de monnaies sociales et leur adéquation à des objectifs spécifiques. Se pose donc la question des conditions de viabilité des dispositifs de monnaies sociales. L'analyse de ces conditions doit impliquer des jugements quant aux formes observées et ceci doit conduire à évaluer de façon pragmatique la validité des dispositifs. Il ne s'agit pas ici de prétendre épuiser l'étendue des débats en la matière, mais néanmoins de poser une partie importante des points qui méritent discussion. Il s'agit là d'un véritable enjeu pour la seconde génération de dispositifs qui émerge depuis le début des années 2000.

Pour discuter de ces questions organisationnelles, on discute d'abord des raisons d'être de ces dispositifs et on part en particulier de l'idée selon laquelle les monnaies sociales visent des objectifs de localisation, de dynamisation et de transformation de la nature des échanges (section 2). On distingue dans la suite de ce texte deux grands domaines dans lesquels des choix sont non seulement possibles mais orientent fortement les dispositifs : d'une part, la taille de ces dispositifs et les catégories de personnes et d'échange (section 3) ; d'autre part, les conditions de l'émission, de la circulation et de la convertibilité des monnaies (section 4). L'exposé des contraintes posées par chacune des modalités d'organisation possibles conduit à clarifier leurs possibilités et leurs limites et à définir cinq modèles de monnaies sociales disposant chacun d'une certaine

1 Le SOL est un projet de monnaie complémentaire destinée à stimuler des activités d'utilité sociale et environnementale. Il est mis en œuvre par le groupe Chèque Déjeuner, le Crédit coopératif, la MAIF et la MACIF et bénéficie du soutien d'un programme européen EQUAL. Il est en phase expérimentale de 2006 à 2008 sur cinq territoires français, sur la base de partenariats avec des collectivités locales. Voir le site http://www.sol-reseau.coop/ 
cohérence relativement à leurs objectifs (section 5).

\section{Rationalités multiples des monnaies sociales}

Comment comprendre l'existence des monnaies sociales ? Avant qu'émerge la vague des monnaies sociales au début des années 1980, aucun texte ne présentait de théorie de ce qui allait être mis en œuvre. En effet, il ne suffit pas de chercher des fondateurs parmi ceux qui ont développé des approches hétérodoxes de la monnaie en promouvant des systèmes monétaires locaux et / ou privés ; il faut aussi s'assurer que les dispositifs mis en place par la suite ont effectivement visé à appliquer ces principes. Ainsi, les approches de free banking ou de dénationalisation de la monnaie à la Hayek ne sauraient être invoquées, bien que l'on trouve l'idée d'une restauration du pouvoir d'émission monétaire hors du contrôle de la sphère publique parmi des penseurs des monnaies sociales (Lietaer, 2001 et surtout Greco, 2001). Il est vrai que l'on peut trouver des traces d'une approche considérée comme utopique (et son auteur comme «monetary crank»), celle de Silvio Gesell (1916), non seulement dans les textes de promoteurs actuels des monnaies sociales (Kennedy, 1990 ; Lietaer, 2001) mais aussi dans les règles effectivement mises en place dans de nombreux dispositifs, sous la forme d'un principe de dépréciation des avoirs (la «fonte » de la monnaie, nous en reparlerons) afin d'inciter à la dépense ; mais une telle référence ne saurait épuiser le phénomène et fournir une explication suffisante étant donné que, la plupart du temps, il s'agit là d'une composante parmi d'autres du système monétaire mis en place. Il faut, enfin, se méfier d'explications ex post et ad hoc plaquant sur les dispositifs nés depuis les années 1980 des rationalités qui n'étaient pas présentes dans leur établissement. L'objet de ce texte n'est pas d'élaborer une théorie des monnaies sociales, mais un détour par les mobiles à l'œuvre dans ces dispositifs permet d'éclairer leurs spécificités.

Il faut introduire une première distinction pour bien comprendre la nature des monnaies sociales. $\mathrm{Ce}$ ne sont pas des monnaies « publiques », au sens où leur définition et leur émission relèveraient d'une logique politique associée à une forme de souveraineté, que celle-ci émane d'une autorité centrale ou fédérale pour les monnaies officielles, ou d'autorités publiques locales, notamment fédérées, pour de nombreux cas de monnaies locales (voir par exemple le cas des monnaies provinciales argentines entre 1984 et 2003 (Théret et Zanabria, 2007)). Ce ne sont pas non plus des monnaies «privées » dont la définition et l'émission relèveraient d'une logique et d'organisations lucratives. Soulignons que, lorsqu'une banque intervient dans ces dispositifs, il s'agit d'une banque coopérative locale (banque Palmas, Fortaleza, Brésil) ou de type credit 
union (Ithaca, Etats-Unis). La plupart du temps, elle ne fait d'ailleurs qu'adhérer à un dispositif initialement imaginé sans elle et n'est pas émettrice de la monnaie comme le serait toute banque en monnaie nationale via son activité de crédit (Ithaca aux Etats-Unis ; Chiemgauer en Allemagne ; SOL en France). Ainsi, par contraste, les dispositifs de monnaies sociales sont créés dans une logique citoyenne par des groupes de personnes qui agissent hors du pouvoir politique (et d'une logique politique) comme du pouvoir économique (et d'une logique lucrative).

Une observation attentive des dispositifs dans leur variété et une lecture des textes écrits par leurs promoteurs permet d'identifier des objectifs spécifiques qui sont assignés aux monnaies sociales. Cela doit permettre de marquer leurs différences à l'égard des monnaies nationales. On peut distinguer trois grands objectifs.

D'abord, il s'agit de localiser les transactions à l'intérieur d'un espace, en privilégiant l'usage local de revenus tirés d'une production locale. Les principales spécificités des monnaies sociales en la matière sont certes le caractère réduit de cet espace, mais surtout sa définition souvent communautaire plutôt que territoriale. Un dispositif comme le CES (Community Exchange System), par exemple², a une prétention aterritoriale (ancré dans une plateforme de gestion Internet, élaboré d'abord au Cap, en Afrique du Sud, il prétend pouvoir être utilisé de façon déterritorialisée voire transnationale du fait de la souplesse de l'outil Internet) ; comme les autres dispositifs de type LETS, il s'agit d'abord d'un réseau de personnes adhérentes, et non d'un espace géographique précisément délimité ; qui plus est, cet espace apparaît «alternatif » à l'économie formelle autant qu'à l'économie informelle (voir, sur ces espaces et géographies particuliers, Hart, 2000 ; Leyshon, Lee et Williams, 2003).

Ensuite, cette localisation doit conduire à une dynamisation des échanges au bénéfice des populations, qui passe par divers dispositifs liés en particulier au crédit et au taux d'intérêt. On trouve là des orientations peu différentes de celles que l'on peut trouver pour d'autres monnaies, si ce n'est leur aspect fréquemment utopique comme en témoigne le recours à une approche telle celle de Silvio Gesell, pourtant reconnu (de façon provocatrice) par Keynes (1936, p. 353), et dont l'idée d'accélération de la circulation monétaire au moyen d'une taxe sur les avoirs monétaires a inspiré Irving Fisher (1933) dans ses propres propositions de «reflation » en 1932-33 pour les Etats-Unis. De façon générale, on trouve là un refus de 
l'accumulation, de la conservation et de la concentration de la richesse, et au contraire une promotion de la circulation de la richesse via une distribution équitable, sinon égale, de la monnaie.

Localisation et dynamisation renvoient à un problème concret auquel font face les fondateurs de dispositifs dans de nombreux cas, particulièrement dans des pays du Sud : le manque de revenus en monnaie de populations pauvres, lié à la faiblesse de l'emploi mais aussi aux insuffisances du système bancaire. La principale réponse à micro-échelle à ces deux problèmes est venue de la microfinance et plus particulièrement du microcrédit, qui approvisionne les populations en monnaie ; une autre réponse, moins connue, moins répandue et ne bénéficiant pas des mêmes facilités, peut venir des monnaies sociales. Alors que le microcrédit fournit des avoirs en monnaie nationale, à rembourser moyennant souvent des taux d'intérêt importants (bien qu'inférieurs aux taux pratiqués par les usuriers traditionnels), et dont l'usage peut être orienté vers des biens produits loin de l'espace local, les monnaies sociales privilégient une fourniture d'avoirs, généralement sans taux d'intérêt, en une monnaie interne dont la convertibilité est coûteuse voire impossible et qui, par conséquent, va orienter la dépense vers des productions locales.

Il s'agit enfin de transformer les pratiques et les représentations de l'échange. Ce dernier objectif distingue spécifiquement les monnaies sociales des autres formes de monnaies locales et par conséquent des autres formes de monnaies en général ; il justifie aussi dans une certaine mesure le qualificatif de « social» accolé à cette forme de monnaie (Blanc, 2007). Cette transformation porte en particulier sur trois domaines. Elle vise à modifier le statut des échangistes, en revalorisant les capacités productives non monnayables des personnes dans le cadre du salariat ou des professions indépendantes : tel est le sens clairement affiché du « prosommateur» dans le trueque argentin (inspiré de Toffler, 1980), cet individu dont la faculté combinée de production et de consommation conditionne l'efficacité du dispositif (Montillet, 2003). La transformation vise aussi la relation qui s'établit entre les partenaires des échanges, en promouvant, par la confiance, le développement de relations interpersonnelles, depuis la convivialité jusqu'à l'amitié. On peut voir là un renversement de la vision économiste de la monnaie, qui en fait d'abord un moyen de paiement, c'est-à-dire un outil qui coupe le lien entre les partenaires d'un échange, au profit d'une vision de la monnaie comme promoteur d'une relation d'échange porteuse de cohésion sociale (Servet, dir., 1999). Enfin, la transformation vise les règles marchandes, en établissant, par exemple, des règles de fixation des prix qui

\footnotetext{
2 Voir http://www.sane.org.za/
} 
éloignent les transactions de la stricte logique de l'échange marchand. Autrement dit, il est souvent proposé que la valeur des biens et services fournis se détache du système de prix en vigueur à l'extérieur, en particulier en imposant, avec plus ou moins de succès, une évaluation des biens et des services échangés en fonction du temps : temps de prestation d'un service ou temps d'élaboration d'un bien, étant entendu qu'il faut aussi tenir compte des intrants nécessaires. Mais ces règles ne sauraient cacher qu'il reste très difficile d'établir et de maintenir une telle distance à l'égard des prix de marché, comme nous le verrons par la suite.

On le voit, les différences, affirmées par leurs promoteurs, des monnaies sociales vis-à-vis d'autres formes de monnaies ne peuvent se ramener à un simple écart dans l'étendue de l'espace de circulation de la monnaie. Nous verrons néanmoins que, au sein de la diversité des modèles existants de monnaies sociales, certains dispositifs tendent à atténuer fortement l'objectif de transformation et se rapprochent de monnaies locales plus classiques, davantage accessibles par une argumentation économique d'ordre théorique.

\section{Taille des dispositifs, catégories de personnes impliquées et types d'échanges privilégiés}

Un premier élément organisationnel concerne la combinaison des types de biens et de services privilégiés, de la diversité des activités présentes dans le dispositif, de la taille de celui-ci (en nombre de partenaires) et de la qualité des partenaires (particuliers et professionnels).

\subsection{Entre trop et trop peu de personnes et d'activités}

L'établissement d'une monnaie locale a mécaniquement deux effets contradictoires. D'une part, les porteurs de la monnaie locale sont conduits à orienter leurs échanges médiatisés par cette monnaie vers des personnes physiques ou morales qui l'acceptent, ce qui contribue à un effet de rapatriement d'échanges à l'intérieur de l'espace local ; d'autre part, le défaut relatif de validité de la monnaie locale (puisqu'elle n'est valide que localement) peut conduire nombre de personnes à refuser de l'employer et donc d'entrer dans la logique du localisme monétaire. Le résultat de ces deux effets cumulés dépend des caractéristiques de la monnaie locale et de celles de la monnaie nationale dans laquelle les échanges habituels ont lieu.

Ce qui peut inciter à l'usage d'une monnaie locale est par exemple le contexte d'une inflation élevée pénalisant la monnaie nationale ; c'est aussi le contexte d'une pénurie de cette monnaie conduisant à trouver dans l'usage d'une monnaie locale un moyen de faire face. Il est indéniable par exemple que, durant les années 2001-2002 en Argentine, la contraction des revenus en peso pour toutes les populations touchées par 
le chômage a conduit à chercher dans les clubs de trueque un moyen de contourner le problème. Lorsque, en 2002, le gouvernement a mis en place un programme d'aide sociale très étendu, les avantages perçus à rester dans ces dispositifs ont fortement diminué, et ce d'autant plus que dans ces dispositifs les nouveaux adhérents n'avaient pas pu, de toute évidence, trouver un palliatif suffisant du défaut de revenus en peso (Luzzi, 2005 ; Montillet, 2006).

Surtout, un nombre insuffisant de personnes, de commerces et de producteurs acceptant cette monnaie est particulièrement dissuasif: que faire d'une monnaie dont l'usage serait trop restreint? L'obtention d'une telle quantité apparaît dès lors comme l'une des conditions nécessaires au maintien et au développement des dispositifs mis en place. Ainsi, un dispositif de faible taille risque fort d'être peu efficace pour localiser les échanges et, en conséquence, risque de donner peu de prise à leur dynamisation.

Or, à partir du moment où l'on intègre des professionnels, artisans et autres producteurs opérant aussi, et en priorité, à l'extérieur du dispositif, l'ampleur des revenus qu'ils obtiennent en monnaie locale doit trouver des contreparties significatives, dont tout ou partie devra être de nature professionnelle aussi. Pour cela, le dispositif doit avoir une taille et une variété minimales relativement importantes, sous peine de voir les professionnels le quitter et de le voir se transformer en dispositif tourné de manière exclusive vers les particuliers. C'est ainsi qu'un des problèmes de l'expérience mise en œuvre en 2002-2003 dans un quartier de Fortaleza, au Brésil, par la banque coopérative locale Palmas et l'ONG Strohalm, a été l'accumulation par certaines entreprises de monnaie locale trop peu utilisable. Quarante entreprises locales avaient formellement accepté d'intégrer le dispositif, alors que les organisateurs souhaitaient à l'origine en convaincre cent ; cela n'a pas suffi, à l'évidence, pour que les entreprises aient un usage satisfaisant de la monnaie locale obtenue de leurs ventes, mais semble avoir suffi aux consommateurs pour avoir le sentiment que cette monnaie disposait d'un pouvoir d'achat suffisamment étendu (Ferreira et Moers, 2006).

Un dispositif uniquement centré sur les particuliers, au contraire, nécessite une taille minimale nettement moins importante. En France, où seuls les particuliers adhèrent aux SEL (qui sont la forme française des LETS), il semble qu'un dispositif concernant moins de trente à quarante personnes actives dans les échanges végète et finalement étouffe. Lorsque des dispositifs de petite taille sont situés dans des zones assez proches, la tentation est grande de fusionner pour redonner du souffle aux échanges.

Une situation intermédiaire peut émerger avec la spécialisation de certains particuliers dans la fourniture 
de prestations ou de biens dans le dispositif mais non à l'extérieur ; ces particuliers semi-professionnalisés, ou semi-professionnels endogènes, vont exiger un éventail d'activités intermédiaire permettant d'obtenir de façon régulière les intrants nécessaires à leur production, sans passer par le commerce courant qui pourrait s'avérer onéreux; dans le même temps, l'usage des revenus tirés de cette activité plus ou moins régulière suppose un éventail de biens et de services plus étoffé que dans le cas de dispositifs centrés sur les seuls particuliers non spécialisés.

\section{2. Échanges et partenaires à éviter}

L'intégration de professionnels est donc exigeante quant aux conditions de localisation et de dynamisation des échanges ; elle l'est aussi quant aux conditions de la transformation des échanges. Le professionnel apporte en effet au dispositif des biens ou des services fournis par ailleurs dans les conditions d'échange courantes. On comprend que des arbitrages puissent se développer tant du côté des professionnels fournissant ces biens ou services que des particuliers y accédant, le tout conduisant à un alignement des conditions de leur fourniture dans le dispositif par rapport aux conditions courantes : retrait des professionnels dès lors qu'il devient moins intéressant de fournir dans le dispositif qu'à l'extérieur, fuite des particuliers pour accéder aux biens et aux services hors du dispositif dès lors qu'aucun avantage n'apparaît à l'intérieur, etc. On a pu observer en Argentine durant la phase de crise aiguë de 2001-2002 la multiplication de revente en interne de produits alimentaires achetés dans les supermarchés par des membres des dispositifs (les nodos). Cette revente leur procurait un revenu en monnaie interne (les creditos) qu'ils utilisaient pour se procurer des biens et services introuvables à l'extérieur ou à des prix trop élevés.

Quoi qu'il en soit, ce type de dérive tend à aligner les prix internes sur les prix externes et le dispositif peut se résumer à une annexe de la société marchande. Tel est le choix de certains dispositifs, tandis que d'autres se refusent formellement à une telle perspective. Les dispositifs de monnaies sociales, dans lesquels on met fortement l'accent sur la nécessaire transformation des modalités de l'échange, sont particulièrement sensibles à ce risque. Car, avec l'alignement des prix et les arbitrages qui le provoquent, pénètrent aussi dans le dispositif des pratiques contradictoires avec certains de leurs objectifs : individualisme calculateur, accumulation, retour en force des valeurs marchandes. La monnaie sociale perd de ses spécificités et devient une simple monnaie locale si on en use comme d'une monnaie courante. Le risque de cet «isomorphisme marchand» (Bowring, 2000) peut conduire à choisir l'exclusion de tout type d'échange par lequel les 
pratiques externes pourraient être importées.

Le développement d'échanges de semi-professionnels endogènes (ces particuliers spécialisés dans une production du fait de la dynamique du dispositif, production destinée uniquement à des débouchés internes) est moins dangereux pour l'orientation d'un dispositif de monnaie sociale, car il n'y a pas de possibilité d'arbitrage entre vendre à l'extérieur et vendre à l'intérieur: écouler des pâtisseries maison au sein d'un dispositif ne signifie pas, en général, que l'on pourrait les écouler à l'extérieur, notamment du fait de contraintes d'ordre légal sur ce type d'activités (professionnalisme, TVA, règles sanitaires...).

La nature des choses échangées a aussi des conséquences sur le comportement possible des personnes. Le degré de substituabilité des biens et des services fournis en interne par des biens et des services accessibles dans l'économie externe est un critère permettant de juger du risque d'opportunisme associé à la possibilité des arbitrages. Plus cette substituabilité est importante, plus ce risque est élevé. Sont particulièrement porteurs de ce risque les produits fournis tant en interne qu'en externe et dont la charge affective est nulle. De fait, il s'agit avant tout de biens, car dans la prestation de services il est aisé de mettre l'accent sur la dimension humaine de la relation d'échange. C'est ainsi que des biens et surtout des services pour lesquels les conditions relationnelles de la fourniture jouent un rôle majeur (conseils personnalisés, qualité du contact, paroles échangées...) ont des chances d'échapper à ce risque : par exemple, la garde d'enfants, une coupe de cheveux, une leçon de langue étrangère et diverses activités dans lesquelles l'interaction concourt à la qualité du produit. Enfin, les biens et services strictement inaccessibles en dehors des dispositifs ou accessibles à des conditions tarifaires jugées prohibitives sont exempts de ce risque : par exemple, l'arrosage des plantes pendant une absence, un coup de main pour un déménagement, etc. : on trouve là un ensemble de services de proximité ou à la personne.

\section{Conditions de l'émission, de la circulation et de la convertibilité des monnaies}

L'organisation des monnaies sociales passe par l'établissement de règles relatives à l'émission des moyens de paiement (donc, notamment, au crédit), à leur circulation (donc, notamment, aux formes monétaires privilégiées) et aux conditions de conversion des avoirs entre les monnaies locale et courante.

\subsection{Quelle forme monétaire adopter ?}

Quelle forme monétaire privilégier? Les moyens de paiement peuvent prendre deux grandes formes, 
celle manuelle (en l'occurrence, des billets ou des bons transmissibles de la main à la main) ou celle scripturale (écriture sur comptes, par le biais de dispositifs variés).

Du point de vue des conditions de l'émission, on peut distinguer avec Pantaleo Rizzo (2000) les monnaies « complémentaires » des monnaies «multilatérales ». Les monnaies dites complémentaires sont émises de façon centralisée par une instance qui décide de l'émission; les échanges interviennent après que l'émission a eu lieu par la distribution de la monnaie aux adhérents (soit par crédit, soit par don, soit enfin par conversion de monnaie nationale en monnaie interne). C'est le cas, par exemple, de l'Ithaca Hour, des réseaux argentins, de la monnaie Palma à Fortaleza, etc. Rien n'interdit le recours à une combinaison de moyens de paiement manuels et scripturaux, sinon la complexité de sa mise en œuvre ; de fait, on observe plutôt le recours à l'une de ces deux formes à l'exclusion de l'autre. Les monnaies dites multilatérales sont émises de façon décentralisée et automatique, selon les besoins de l'échange : ce sont les échanges qui, en nécessitant leur comptabilisation, entraînent une émission monétaire. Ceci interdit le recours à de la monnaie manuelle. Ce sont des systèmes de monnaie scripturale à solde global nul, les débits des uns compensant les crédits des autres. Ils sont généralement dotés de limites (les soldes ne pouvant dépasser un certain montant en valeur absolue) afin d'éviter les dérives comme la captation au bénéfice de quelques-uns de la dynamique des échanges. L'exemple fondateur est celui des multiples formes des local exchange and trading systems (LETS).

Tableau 1 - Diversité des formes de monnaie dans les dispositifs de monnaies sociales

\begin{tabular}{|l|l|l|}
\hline & Forme manuelle & Forme scripturale \\
\hline $\begin{array}{l}\text { Monnaie } \\
\text { « complémentaire » }\end{array}$ & $\begin{array}{l}\text { Forme dominante dans les monnaies } \\
\text { « complémentaires » } \\
\text { Ithaca Hour (Etats-Unis), trueque } \\
\text { (Argentine), Palma (Fortaleza, Brésil), } \\
\text { Chiemgauer (Allemagne) }\end{array}$ & $\begin{array}{l}\text { Forme peu fréquente } \\
\text { Projet SOL (France) }\end{array}$ \\
\hline $\begin{array}{l}\text { Monnaie } \\
\text { «multilatérale » }\end{array}$ & $\begin{array}{l}\text { Forme subordonnée, employée dans } \\
\text { une phase particulière de la vie du } \\
\text { LETS / SEL }\end{array}$ & $\begin{array}{l}\text { Forme dominante dans les monnaies } \\
\text { « multilatérales » } \\
\text { LETS, dont les SEL français ; } \\
\text { Community Exchange System (Afrique } \\
\text { du Sud) }\end{array}$ \\
\hline
\end{tabular}

Le choix d'une forme monétaire n'est pas sans incidences sur l'organisation du dispositif : tout d'abord, chaque forme monétaire a ses propres coûts ; ensuite, chacune peut requérir des modalités d'émission, donc de crédit, particulières. 
Les systèmes totalement scripturaux ont d'abord été coûteux car la comptabilisation des échanges et la gestion des comptes étaient réalisées à la main : réception de la partie des bons d'échanges destinée à l'administration ${ }^{3}$, inscription sur un livre de compte ou sur une feuille de compte informatique, etc. Ce coût est d'autant plus élevé que le dispositif est étendu : ainsi la gestion des comptes des quelque 2000 membres du LETS australien de Katoomba a occupé jusqu'à une vingtaine de personnes dans les années 1990.

Depuis le début des années 2000, la diffusion très large d'Internet et des technologies mobiles a permis de développer des systèmes permettant aux utilisateurs eux-mêmes d'opérer les démarches de comptabilisation des échanges, via une plateforme accessible par Internet (laquelle peut être couplée à un système de transmission par téléphone mobile) : tel est le cas du Community Exchange System (CES) développé en Afrique du Sud.

Avant, cependant, que ces possibilités émergent, la lourdeur de la gestion manuelle centralisée des monnaies sociales scripturales a pu conduire à lui préférer la monnaie manuelle, ne nécessitant aucune tenue centralisée des comptes personnels : soit dans certaines phases de la vie d'un dispositif qui demeure scriptural (c'est ainsi que, au début des bourses locales d'échange - BLE - organisées dans beaucoup de SEL français, des bons sont distribués aux participants, sachant que de nombreux échanges vont avoir lieu en un temps et un espace réduits), soit en organisant l'ensemble du dispositif autour de monnaie manuelle. C'est le choix réalisé dès son origine au sein de l'Ithaca Hour (États-Unis), au début des années 1990 (Glover, 1995). C'est aussi le choix réalisé assez vite par les fondateurs du trueque argentin, après une première phase de fonctionnement scriptural, en 1995, où a été fait le constat de la lourdeur de la comptabilisation des échanges.

Un système de monnaie manuelle évacue le problème de la tenue centralisée des comptes, mais il peut aussi être coûteux. Imprimer des billets implique en effet des coûts que la crainte des falsifications rend encore plus élevés. Cette crainte est d'autant plus forte et justifiée que le système est étendu. Le système argentin a été submergé, vers 2001-2002, de fausse monnaie (dans une proportion que certains disaient proche de 80 à $90 \%$ de la masse monétaire), et les réactions subséquentes ont consisté, du point de vue technique, à imprimer des billets de plus grande qualité avec des systèmes de protection élaborés (Montillet,

\footnotetext{
${ }^{3}$ Dans la plupart des LETS, dont les SEL, leur forme française, les partenaires d'un échange remplissent un bon en trois volets mentionnant en particulier le montant de la transaction : un volet par personne pour mémoire, plus un volet
} 
2003). Les billets en réserve peuvent aussi être volés, ce qui est arrivé, par exemple, à Ithaca. Vol et contrefaçon sont strictement impossibles dans le cadre d'un système purement scriptural et multilatéral de type LETS, puisqu'il n'existe ni billets, ni chèques, ni cartes qui permettraient de voler des avoirs en monnaie interne ${ }^{4}$. La seule fraude possible est l'accumulation de crédits négatifs sans jamais compenser cette dette - les déboires du LETS canadien de Comox Valley en 1985 en sont l'exemple fondateur; ils justifient l'établissement de garde-fous comme des limites maximales de soldes à ne pas dépasser.

Ces coûts liés à la forme monétaire promue peuvent être pris en charge par la collectivité elle-même : pour les systèmes scripturaux, sous la forme du versement d'une contrepartie en monnaie interne aux personnes qui assurent la comptabilité ; pour les systèmes à billets, sous la forme d'une contrepartie publicitaire aux entreprises qui impriment des billets ou d'un paiement direct financé par les cotisations des adhérents en monnaie nationale. Enfin, une possibilité de couvrir un débit laissé par un ancien adhérent indélicat dans un dispositif de monnaie multilatérale consiste à imputer la dette à l'ensemble des adhérents, autrement dit en faire supporter une petite partie par chacun.

D'un point de vue économique, une monnaie multilatérale, c'est-à-dire un système de monnaie scripturale à solde global nul comme le sont les LETS, a l'avantage de confondre création monétaire et besoin de monnaie : la création monétaire a lieu au moment du paiement des échanges, si leur montant dépasse le montant créditeur du solde du payeur. Dans un système à billets, ceux-ci doivent être dans les mains des demandeurs pour qu'un échange puisse avoir lieu ; cela pose la question de l'émission monétaire et par conséquent de ses modalités, de ses limites, de ses contraintes : à quelle occasion émettre de la monnaie, pour qui, chargée d'un taux d'intérêt ou non, en quelle quantité, etc. : toutes questions inutiles dans un système de type LETS dès lors que l'association elle-même ne se fait pas débitrice pour engager des dépenses d'intérêt général. Car, si l'association adopte cette position, des questions par nature politiques, et par conséquent sujettes à discussions voire à tensions, apparaissent : celles des types de dépenses à réaliser, de la notion même d'intérêt général et de la quantité de monnaie à affecter à cela. Curieusement, les LETS, qui sont de formidables outils de construction de liens sociaux et d'apprentissage d'une forme de citoyenneté

destiné à l'administration pour la comptabilisation de l'échange.

${ }^{4}$ Ainsi, la question innocente «Et vous n'avez pas peur que quelqu'un parte avec la caisse? », posée dans les premiers temps des SEL en France par des administratifs intrigués par ces dispositifs et quelque peu soupçonneux, est dénuée de sens. 
par le biais de discussions et de débats relatifs aux règles du jeu internes au groupe, semblent aussi fréquemment construits sur l'expulsion des débats politiques relatifs à de telles actions d'intérêt général... On peut conclure que les monnaies locales à émission monétaire centralisée, c'est-à-dire les monnaies complémentaires au sens de Rizzo (2000), sont plus politiques a priori que les monnaies multilatérales, car la question du contrôle et des modalités de l'émission monétaire se pose systématiquement.

\subsection{Comment gérer l'évolution de la masse monétaire?}

Ainsi que le souligne Stephen DeMeulenaere (2004), la gestion de l'émission et du retrait de monnaie est un point essentiel pour assurer la viabilité d'un système. Or la plupart des dispositifs ne sont pas dotés des outils permettant de gérer correctement la masse monétaire interne. DeMeulenaere souligne par ailleurs l'incompatibilité a priori entre l'objectif de gestion précise de la masse monétaire et l'objectif de simplicité organisationnelle.

Cette gestion dépend d'abord de la forme monétaire adoptée, puisque dans un système de monnaie multilatérale la gestion de la masse monétaire est proprement automatique : la monnaie est simultanée à l'échange. En revanche, dans les dispositifs de monnaie complémentaire, le problème se pose des conditions de l'émission et du retrait. Une solution simple consiste à émettre la monnaie à l'adhésion : tout nouvel adhérent reçoit une somme forfaitaire en monnaie interne qui lui permet de commencer à échanger. Par la suite, c'est au fil de ses échanges que son portefeuille de monnaie interne évoluera. C'est ce qui a été choisi dans le dispositif d'Ithaca, à côté d'autres modalités comme le crédit, que l'on examinera plus loin. C'est ce qui a été choisi dans les dispositifs de trueque argentins, à l'exclusion de toute autre modalité.

Le cas argentin, particulier mais exemplaire, a montré que ce modèle simple manque singulièrement de moyens de régulation (voir notamment Montillet, 2006). L'absence de mécanisme efficace de retrait de la monnaie émise à l'adhésion suffit à le disqualifier. En logique, il faudrait en effet qu'un adhérent qui se retire de l'association rende la monnaie obtenue à l'adhésion, de sorte que la proportion entre la masse monétaire interne et le nombre d'adhérents demeure stable. De fait, ce mécanisme n'a pas fonctionné et il apparaît difficilement applicable en pratique : lorsqu'une personne se retire, elle dépense ses derniers avoirs (les creditos) et ne vient plus, tout simplement ! Au lieu d'être retirée, la monnaie émise est donc réinjectée. Si d'ailleurs un mécanisme de retrait de ce type était efficace, rien ne garantirait pour autant que la masse monétaire distribuée via les adhésions serait correctement proportionnée aux besoins internes. Selon les 
circonstances (c'est-à-dire selon les capacités productives et les besoins de consommation de chacun), une même quantité de monnaie par personne peut se révéler insuffisante ou au contraire trop abondante ${ }^{5}$. Le cas argentin cumule avec ce défaut originel des circonstances qui ont pesé lourdement. On a laissé les personnes adhérer à plusieurs dispositifs simultanément et, par là, obtenir davantage de monnaie interne que celle fournie par une seule adhésion, sans que la capacité productive de ces personnes puisse croître en conséquence. On a laissé, en 2001-2002, des personnes intégrer massivement les dispositifs sans s'assurer que chaque nouvel adhérent était apte à combiner une demande et une capacité productive ; cela a contribué à déséquilibrer l'offre et la demande. La pression sociale à l'adhésion, due à la tournure catastrophique prise par l'économie argentine, a fait sauter le principal verrou qui assurait la stabilité interne du trueque, à savoir les quelques heures nécessaires de formation («capacitación ») qui permettaient de diffuser et de reproduire les valeurs et principes fondateurs des réseaux. La contrefaçon massive dénoncée par les créateurs du principal réseau, la Red Global de Trueque (RGT), a ajouté aux déséquilibres qui ont conduit à une inflation terrible et cette dernière a non seulement fait perdre une grande part de sa valeur au credito $^{6}$ mais aussi décrédibilisé les dispositifs de trueque aux yeux de nombreux Argentins.

Une façon de contenir les risques liés au principe d'émission monétaire à l'adhésion consiste à appliquer des principes inspirés de l'approche de Silvio Gesell (1916). Dans le cadre théorique d'un socialisme de marché inspiré de Proudhon, Gesell proposait de taxer non pas ceux qui ont besoin d'argent (et qui, par là, demandent des crédits, la taxe prenant la forme du taux d'intérêt), mais ceux qui disposent d'argent et qui ne l'utilisent pas. C'est ainsi que le détenteur de monnaie devrait, dans ce cadre, s'acquitter d'une taxe mensuelle de, par exemple, $0,5 \%$ sur la monnaie inemployée, soit, additionnée, $6 \%$ sur l'année 7 . Appliqué dans une vingtaine de localités dans le monde entre les années 1930 et les années 1960 (mais non appliqué comme élément d'une réforme monétaire globale, comme il le proposait), cela se traduisait en particulier par la nécessité d'acheter des timbres à coller au dos des billets dont la valeur, sans cela, fondait à dates

\footnotetext{
${ }^{5} \mathrm{Ce}$ raisonnement vaut toutes choses égales par ailleurs, c'est-à-dire à vitesse de circulation égale ; mais la chute brutale ou, au contraire, l'accélération de la vitesse de circulation de la monnaie est le signe d'une inadéquation entre la quantité de monnaie et les besoins, et il reste à prouver que les changements dans cette même vitesse de circulation parviennent à satisfaire pleinement ces besoins.

${ }^{6}$ Cette dépréciation a été si forte que la RGT a dû par la suite changer son unité de compte : elle a instauré l'unité de compte « trueque » valant 100 creditos et a libellé les nouveaux bons en multiples de cette nouvelle unité de compte.

${ }^{7}$ C'est un exemple de taux que propose Gesell.
} 
régulières (Onken, 1997 ; Blanc, 1998) ${ }^{8}$. Cette façon de procéder constituait une incitation à dépenser son argent plutôt qu'à acquitter la taxe, le résultat étant une dynamisation de l'activité économique par une meilleure circulation de l'argent. Dans la vague contemporaine des monnaies sociales, des formes de taxation ont été mises en œuvre pour inciter à la dépense. On trouve aujourd'hui deux types d'applications. La première consiste à imposer à intervalle régulier une taxe sur les avoirs inemployés, assez proche des propositions geselliennes. L'incitation à la dépense est donc constante, en général mensuelle. La seconde consiste à imposer une date limite d'utilisation des billets ou des avoirs détenus. Lorsque cette date est atteinte, les avoirs et les billets sont annulés. L'incitation à la dépense est donc moins régulière mais particulièrement puissante, puisque des billets ou des avoirs accumulés deviennent, du jour au lendemain, inutilisables. Dans le réseau argentin RGT, une combinaison de ces deux possibilités est désormais mise en œuvre: la validité des bons est annuelle et leur échange pour de nouveaux bons a lieu moyennant une oxydation de $12 \%$. Cette méthode fournit un outil intéressant de régulation de la masse monétaire qui, de la sorte, ne peut plus s'accumuler.

En conclusion, le principe de l'émission monétaire à l'adhésion, et par conséquent proportionnée au nombre de membres, n'est pas un choix optimal, même s'il a le mérite de la simplicité car il est universellement applicable. Pour régler au mieux la circulation monétaire et contrôler à la fois l'émission et le retrait de monnaie interne, il faut envisager une organisation plus complexe passant par un système de crédit interne, des mécanismes de retrait (par exemple par l'imposition d'une limite de validité de la monnaie dans le temps) et éventuellement la possibilité de convertir des avoirs monétaires depuis l'extérieur vers l'intérieur, et inversement. Ces organisations plus complexes font l'objet de réflexions avancées dans le cadre d'une ONG telle que Strohalm (van Arkel, Ramada et Vink, 2006).

\subsection{Quelle convertibilité des avoirs?}

Dans les dispositifs de monnaie sociale comme dans tout espace monétaire, les flux d'entrée et de sortie de monnaie font varier la masse monétaire. Les choix relatifs à la convertibilité des avoirs sont donc très importants en ce qu'ils conditionnent l'évolution de la masse monétaire. D'un strict point de vue monétaire, donc en faisant abstraction des structures productives, les objectifs de localisation et de dynamisation des

\footnotetext{
${ }^{8}$ C'est ainsi qu'en français on parle souvent de «monnaie fondante ». En anglais, le terme consacré est «demurrage », en référence à une taxe portuaire sur les bateaux immobilisés ; en espagnol, on parle plutôt de « oxidación ».
} 
échanges sont d'autant plus assurées que les avoirs en monnaie locale sont difficilement convertibles en d'autres formes monétaires, de sorte que des revenus gagnés en monnaie locale ne puissent être convertis en une autre monnaie et employés hors de l'espace, communautaire ou territorial, dans lequel on cherche à localiser les échanges.

De façon générale, les monnaies multilatérales, de par l'organisation même de l'émission monétaire, interdisent toute entrée comme toute sortie de monnaie. La chose est possible, en revanche, pour les monnaies complémentaires. Il faut bien ici distinguer ce qui relève des possibilités pour une personne, conduisant à une circulation et à un échange de monnaies, et ce qui relève des possibilités du système en général, conduisant à une émission ou à un retrait de monnaie interne. Si par exemple dans le cas argentin des personnes ont pu, de façon clandestine (car non autorisée dans le cadre des règles internes) changer des pesos en creditos ou des creditos en pesos, au moyen d'un taux de conversion variable, cette opération n'a produit qu'une circulation de monnaies et non une émission ou un retrait. Au niveau des clubs et des réseaux de trueque, aucune émission et aucun retrait n'ont pu avoir lieu (sauf d'une façon alors frauduleuse par des organisateurs indélicats) via la conversion de pesos en creditos ou de creditos en pesos.

L'inconvertibilité des avoirs contraint les personnes à ne les employer que pour de la dépense interne. Elle favorise donc la localisation des échanges et leur dynamisation, mais établit dans le même temps un coût d'opportunité à l'entrée dans le dispositif, d'autant plus élevé que les nouveaux entrants doivent cotiser, à la fois pour obtenir un équivalent en monnaie interne et pour alimenter les caisses de la structure permettant de financer ses activités (achat de matériel, fournitures courantes, loyer d'un local, impression de billets, etc. $)^{9}$. Les mobiles socio-économiques de la monnaie locale (c'est-à-dire les raisons qui sont au cœur de sa mise en œuvre, renvoyant à des rationalités économiques ou à des dimensions davantage idéologiques) doivent donc être d'autant plus fortement affirmés pour inciter à l'entrée. Cette barrière peut aussi fonctionner comme mécanisme de sélection des personnes et des comportements en éliminant les logiques opportunistes. À ce titre, l'inconvertibilité des avoirs rend plus solide le dispositif de monnaie locale qui s'appuie sur elle dès lors que ce dispositif cherche à construire un espace socio-économique relativement autonome (SEL français, time banks britanniques, banques du temps italiennes...); en revanche, elle peut être rédhibitoire pour les dispositifs qui visent centralement à s'insérer dans le tissu socio-économique local 
et le dynamiser.

C'est ainsi que certains dispositifs, de type monnaies complémentaires, autorisent les transferts d'avoirs dans le sens de l'entrée et, plus rarement, dans les deux sens de l'entrée et de la sortie. Le dispositif de l'Ithaca Hour, par exemple, autorise l'émission de « hours » contre des dollars (à raison de 10 dollars pour 1 hour), auprès de l'instance qui sert d'institut d'émission (la librairie Autumn Leaves). La conversion inverse, qui se traduirait par une réduction de la masse monétaire interne, n'est en revanche pas possible ${ }^{10}$. Les projets de monnaies locales allemandes Regio autorisent l'entrée comme la sortie, mais imposent à la sortie une taxe de $5 \%$ sur le montant de monnaie interne à changer en euro afin de limiter les sorties (Kennedy, 2006). Une taxe à la sortie était aussi un choix réalisé sur les dispositifs de monnaie locale fondante qui ont été organisés en France dans les années 1950 (Onken, 1997). Lorsque la sortie est possible, même taxée, une contrainte forte apparaît : celle de la couverture, intégrale ou proche de l'intégralité, des émissions de monnaie interne par des réserves accumulées en monnaie externe. On comprend par là que la logique est fondamentalement différente de dispositifs qui surajoutent, même d'une façon limitée, à la circulation monétaire classique une circulation de monnaie sociale. Une conséquence notable est l'impossibilité, pour les dispositifs autorisant les transferts, d'établir de façon crédible un système de valeurs différent visant à hiérarchiser les prix différemment et à se déconnecter ainsi du système de prix en vigueur dans le reste de l'économie. C'est ainsi par exemple que l'unité de compte du dispositif d'Ithaca, le hour, ou « heure », est une fiction au sens où la règle qui domine est sa parité fixe avec le dollar : dix dollars pour une heure. Derrière le compte en heures, il y a le compte en dollar. Au contraire, dans des dispositifs interdisant tout transfert et développant des échanges sur des biens ou des services inaccessibles par ailleurs, une hiérarchie alternative des valeurs est bien davantage crédible.

\subsection{Qui accède à la monnaie ?}

La forme monétaire promue est aussi liée à la façon dont la monnaie doit pouvoir circuler. On peut distinguer deux grands types de monnaies sociales : ceux dans lesquels on ne peut accéder à la monnaie et aux échanges internes qu'après avoir fait acte d'adhésion, et ceux dans lesquels toute personne, adhérente ou non, peut accéder à la monnaie interne dans le cadre habituel de ses échanges.

\footnotetext{
${ }^{9}$ D'une façon certes différente, cette cotisation apparaît dans beaucoup de SEL français (tous ceux en tout cas qui ont un statut selon la loi de 1901), dans l'Ithaca Hour et dans la Red Global de Trueque argentine par exemple.
} 
Dans le premier cas, où il faut adhérer pour échanger, on construit un système clos où les entrées sont potentiellement filtrables, et où du moins l'acte volontaire de l'adhésion constitue un élément de sélection. Cela ne signifie pas que les monnaies scripturales ne puissent se diffuser largement, comme l'illustre la grande taille de certains dispositifs (on a parlé plus haut de celui australien de Katoomba) ; mais la contrainte de l'adhésion fait que toute personne supplémentaire recourant à la monnaie locale se reconnaît formellement dans les valeurs et les objectifs de l'association. La monnaie scripturale apparaît comme la forme monétaire adaptée permettant d'éviter toute diffusion de ce signe communautaire au-delà de la communauté. Les monnaies multilatérales des LETS sont précisément dans ce premier cas, même si dans certaines situations (en France, les BLE, bourses locales d'échange) des bons peuvent être momentanément distribués, dans le cadre d'échanges localisés dans le temps et dans l'espace d'une petite foire.

L'établissement d'un système scriptural verrouillant le périmètre des utilisateurs de la monnaie peut être atténué par l'établissement de passerelles avec des dispositifs voisins en institutionnalisant les échanges (par exemple ceux dits «inter-LETS ») ou en développant des échanges spécifiques concernant par nature des membres de dispositifs différents (par exemple, la Route des SEL en France fonctionne sur la base des membres de SEL différents au travers du pays $\left.{ }^{11}\right)$.

Dans le second cas, où accéder à la monnaie interne ne requiert pas d'adhésion, les utilisateurs de la monnaie locale sont beaucoup plus divers et peut y venir toute personne ne se réclamant pas forcément de la logique et des règles établies par ailleurs. On comprend que le pouvoir de diffusion d'une monnaie locale de ce type est beaucoup plus important, même s'il est corrélé avec un risque important lui aussi de dissolution des valeurs et des objectifs initiaux au fil de son extension. Ce pouvoir de diffusion important a été l'un des facteurs motivant les fondateurs de l'Ithaca Hour à éviter le système scriptural des LETS au profit d'un système de monnaie manuelle (Glover, 1995 ; Douthwaite, 1996). L'Ithaca Hour fonctionne sur la base d'adhérents, particulier comme professionnels. Ils obtiennent de la monnaie interne en changeant du dollar ; en plus de cela, les entreprises qui s'annoncent dans le catalogue d'activités diffusé aux membres reçoivent régulièrement des hours comme récompense de leur fidélité, ce qui concourt à l'émission monétaire interne. Enfin, des organisations obtiennent des crédits en hour. Ces adhérents constituent la base des utilisateurs de la monnaie interne mais, comme monnaie manuelle transmissible de la main à la main, son emploi est ouvert

10 Voir le site http://www.ithacahours.org 
à tous. C'est le pari de dispositifs ouverts visant une dynamisation de l'activité économique locale davantage que l'intensification des liens sociaux au moyen des échanges internes.

\subsection{Du crédit personnel au crédit d'investissement}

Moins directement confrontés à des questions «politiques » fortes relatives au crédit, les dispositifs à crédit automatique et limité comme les LETS sont aussi moins ambitieux. Ici, le crédit n'est pas un crédit d'investissement, il n'est pas la validation sociale d'un projet personnel. C'est un crédit d'accès (pour ne pas dire de consommation), ou encore un crédit personnel dans toutes ses acceptions ${ }^{12}$ : une confiance placée dans la responsabilité des personnes à compenser ultérieurement leur crédit par l'acte de fournir à autrui des biens ou des services.

Les monnaies locales gagnent en ambition socio-économique lorsqu'elles incluent la possibilité du crédit d'investissement, c'est-à-dire du crédit pour des porteurs de projets d'activité. De tels crédits ont été expérimentés dans l'Ithaca Hour, mais ce n'était pas le cœur de l'initiative lors de sa mise en œuvre au début des années 1990. Rares sont, jusqu'ici, les dispositifs de monnaie locale qui visent explicitement le développement du crédit aux porteurs de projets. Le cas de la banque Palmas à Fortaleza (Brésil) demeure isolé (Ferreira et Moers, 2006). La logique de localisation et de dynamisation s'en trouve considérablement renforcée, car ce n'est plus seulement une solidarité d'échanges réciproques qui est promue mais un développement local solidaire. Un micro-entrepreneuriat enraciné localement et porteur de valeurs de réciprocité émerge, qui peut être vu comme la raison d'être de ces monnaies locales. Deux raisons principales expliquent que ce type de dispositif demeure rare. D'une part, il suppose une technicité certaine qui, à Fortaleza, se traduit par le recours à une banque coopérative locale (et, pour l'expérimentation ponctuelle analysée par Ferreira et Moers, 2006, un partenariat avec une ONG développant une triple capacité d'expertise, d'expérimentation et de levée de fonds). D'autre part, ce dispositif met moins l'accent que d'autres sur le motif de transformation de la nature des échanges et privilégie un objectif de développement local solidaire, plus exigeant au plan économique et donc moins susceptible d'être porté directement par des groupes de citoyens peu organisés préalablement. Ce type de dispositif suppose au contraire un certain degré d'organisation préalable : à Fortaleza, le projet de monnaie locale est issu du

11 Voir le site http://selidaire.org/spip/ 
travail déjà ancien de l'association pour le développement du quartier, l'ASMOCONP, créée en 1981, ellemême à l'origine de la création de la banque coopérative locale Palmas en 1998 qui, peu après, a porté le projet de monnaie locale.

On en revient dès lors à la question de l'intégration de professionnels dans les dispositifs. La taille minimale requise pour que ceux-ci soient efficaces dans leur fonction de localisation et de dynamisation des échanges est accrue. La microfinance ouvre les monnaies locales à d'autres horizons dans lesquels ce qui peut apparaître comme le folklore d'un retour de convivialité (ainsi qu'on peut parfois percevoir les SEL français) cède la place à une logique forte de développement local solidaire (ainsi que l'on peut percevoir le projet Palma). Mais alors l'étanchéité du dispositif à l'égard de l'environnement socio-économique devient un obstacle. Il faut au moins permettre que la monnaie locale et la monnaie nationale soient employables simultanément, dans des échanges où les charges externes (fournisseurs externes, taxes diverses) peuvent être assumées par le paiement d'une partie du prix en monnaie nationale (cas de l'Ithaca Hour, et du Palma). Par ailleurs, cette ouverture suppose l'usage de moyens de paiement utilisables aisément, de façon décentralisée et par qui que ce soit sans l'exigence d'un acte volontaire d'adhésion. Il est impossible dans ces conditions de confiner l'organisation monétaire interne à une stricte monnaie scripturale. Enfin, on peut concevoir la possibilité de transferts d'avoirs entre monnaie locale et monnaie nationale, en y apportant quelques freins de principe par le biais de taxes sur la conversion des avoirs.

\section{Types de dispositifs et impact socio-économique}

La discussion de cet ensemble de contraintes organisationnelles conduit à clarifier les possibilités et les limites des dispositifs de monnaies sociales. Ils ont à arbitrer entre, d'un côté, une grande variété de biens et de services qui est associée à l'inclusion de partenaires professionnels et présente dès lors un risque de dissolution d'objectifs de transformation de la nature des échanges et, de l'autre, une taille et une diversité des échanges réduites, présentant au contraire un risque de constituer un groupuscule incapable d'attirer de nouveaux membres. L'éloignement du marché est d'autant plus fort que la monnaie est peu convertible, que les professionnels sont absents et que les dispositifs ont une petite taille. Les dispositifs de monnaies sociales ont aussi à arbitrer entre des règles monétaires qui permettent à tout un chacun d'accéder facilement aux

12 Symptomatiquement, Rizzo (2000) parle dans ce cas de «monnaie multilatérale» mais aussi de «monnaie personnelle ». 
monnaies sociales, présentant le risque d'une dissolution des objectifs, et des règles monétaires strictes qui ferment le système aux seuls membres adhérents et établissent un cloisonnement étanche à l'égard des flux monétaires externes, présentant un risque de stagnation.

Cette discussion permet ainsi d'identifier des types de dispositifs en fonction de critères organisationnels dont on peut évaluer la cohérence. Le tableau 1 présente cinq modèles, que l'on peut rapprocher de dispositifs existants : en particulier, les SEL français pour le modèle 1 « Entraide et convivialité », les LETS anglo-saxons pour le modèle 2 «Couverture de besoins de consommation », le trueque argentin pour le modèle 3 «Valorisation des compétences », le projet SOL pour le modèle 4 «Stimulation de pratiques solidaires» (Delille et Whitaker, 2006), le palma de Fortaleza pour le modèle 5 «Développement local solidaire ». Cette typologie ne prétend pas à l'exhaustivité et l'on doit pouvoir observer des formes intermédiaires. Cependant les croisements entre ces types apparaissent difficiles et leur viabilité est incertaine. Par exemple, l'emploi d'une monnaie multilatérale, nécessairement scripturale, apparaît logiquement incompatible avec les modèles 4 et 5 alors qu'il apparaît logiquement en concordance avec le modèle 1. Les choix organisationnels ne sont pas anodins et déterminent à la fois les objectifs atteignables et la validité des dispositifs.

L'impact socio-économique de ces dispositifs semble croissant au fur et à mesure que l'on évolue depuis le premier modèle en direction du cinquième. Les deux derniers sont les plus exigeants en termes d'ingénierie, car ils imposent de délaisser l'amateurisme qui domine encore dans les autres modèles pour entrer de plain-pied dans un processus gestionnaire où prime l'efficacité. Ils sont exigeants aussi, car ils intègrent davantage d'acteurs. Le dernier modèle demande quant à lui à être couplé avec une banque ; les difficultés liées à cet ensemble d'exigences semblent à la hauteur de ses potentialités. 
Tableau 2. Cinq types de monnaies sociales

\begin{tabular}{|c|c|c|c|c|c|}
\hline Modèle & 1. Entraide et convivialité & $\begin{array}{l}\text { 2. Couverture de besoins de } \\
\text { consommation }\end{array}$ & 3. Valorisation des compétences & $\begin{array}{l}\text { 4. Stimulation de pratiques } \\
\text { solidaires }\end{array}$ & 5. Développement local solidaire \\
\hline Exemples proches & $\begin{array}{c}\text { SEL français, Time banks } \\
\text { britanniques, Banche del tempo } \\
\text { italiennes }\end{array}$ & $\begin{array}{c}\text { LETS anglo-saxons, Talent } \\
\text { germaniques, Tauschringe } \\
\text { allemands }\end{array}$ & Trueque argentin & Projet Sol, projet Libra & $\begin{array}{l}\text { Palma à Fortaleza (Brésil), Regio } \\
\text { du Chiemgau (Allemagne) }\end{array}$ \\
\hline $\begin{array}{l}\text { Principal objectif } \\
\text { pertinent }\end{array}$ & $\begin{array}{l}\text { Socialisation : réinsérer l'échange } \\
\text { dans le lien social }\end{array}$ & $\begin{array}{l}\text { Couverture complémentaire de } \\
\text { besoins de consommation finale }\end{array}$ & $\begin{array}{l}\text { Valorisation des capacités } \\
\text { productives personnelles }\end{array}$ & $\begin{array}{l}\text { Dynamisation d'activités } \\
\text { spécifiques sans but lucratif }\end{array}$ & $\begin{array}{l}\text { Développement d'un tissu local de } \\
\text { micro-entrepreneurs éduqués à } \\
\text { l'autonomie et à la solidarité locales } \\
\text { et développement d'infrastructures } \\
\text { collectives }\end{array}$ \\
\hline $\begin{array}{l}\text { Mode de construction } \\
\text { de la solidarité }\end{array}$ & Réciprocité des échanges & $\begin{array}{l}\text { Réciprocité de la satisfaction de } \\
\text { besoins }\end{array}$ & $\begin{array}{l}\text { Valorisation des compétences de } \\
\text { chacun }\end{array}$ & $\begin{array}{l}\text { Développement d'activités } \\
\text { collectives, solidaires et } \\
\text { d'entreprises sans but lucratif }\end{array}$ & Développement local \\
\hline Usagers & Particuliers & $\begin{array}{l}\text { Particuliers et professionnels } \\
\text { exogènes intervenant en interne }\end{array}$ & $\begin{array}{c}\text { Particuliers et semi-professionnels } \\
\text { endogènes }\end{array}$ & $\begin{array}{l}\text { Particuliers, professionnels et } \\
\text { collectivités publiques }\end{array}$ & $\begin{array}{c}\text { Particuliers, professionnels } \\
\text { endogènes visant aussi une activité } \\
\text { externe, professionnels exogènes } \\
\text { développant aussi une activité } \\
\text { interne }\end{array}$ \\
\hline $\begin{array}{l}\text { Variété minimale } \\
\text { d'activités requise et } \\
\text { taille minimale requise } \\
\text { (nombre d'adhérents) }\end{array}$ & Faibles & Moyennes à fortes & Moyennes à fortes & Fortes à très fortes & Fortes \\
\hline $\begin{array}{l}\text { Spécialisation } \\
\text { productive }\end{array}$ & $\begin{array}{l}\text { Faible, déterminée par les hobbies } \\
\text { et les compétences initiales } \\
\text { acquises à l'extérieur }\end{array}$ & $\begin{array}{l}\text { Faible pour les particuliers et forte } \\
\text { pour les professionnels exogènes } \\
\text { intervenant en interne }\end{array}$ & $\begin{array}{c}\text { Forte, déterminée par les } \\
\text { compétences mises en valeur; } \\
\text { émergence de semi-professionnels } \\
\text { endogènes travaillant en interne } \\
\text { uniquement }\end{array}$ & $\begin{array}{l}\text { Très forte (dans le cadre des } \\
\text { activités existantes) }\end{array}$ & $\begin{array}{l}\text { Très forte, déterminée par les } \\
\text { activités initiales des professionnels } \\
\text { exogènes et par les compétences } \\
\text { orientant le projet des semi- } \\
\text { professionnels endogènes }\end{array}$ \\
\hline $\begin{array}{l}\text { Capacité de } \\
\text { rémunération salariale } \\
\text { en monnaie interne }\end{array}$ & Impossible & Impossible & $\begin{array}{c}\text { Non, car les producteurs sont } \\
\text { individuels : possibilité de filières } \\
\text { productives mais pas d'entreprises } \\
\text { collectives }\end{array}$ & $\begin{array}{l}\text { Oui, mais comme partie marginale } \\
\text { des rémunérations }\end{array}$ & $\begin{array}{l}\text { Oui, comme partie seulement de } \\
\text { salaires aussi versés en monnaie } \\
\text { externe }\end{array}$ \\
\hline
\end{tabular}


Tableau 3. Cinq types de monnaies sociales (suite)

\begin{tabular}{|c|c|c|c|c|c|}
\hline Modèle & 1. Entraide et convivialité & $\begin{array}{l}\text { 2. Couverture de besoins de } \\
\text { consommation }\end{array}$ & 3. Valorisation des compétences & $\begin{array}{l}\text { 4. Stimulation de pratiques } \\
\text { solidaires }\end{array}$ & 5. Développement local solidaire \\
\hline $\begin{array}{l}\text { Type de crédit, de } \\
\text { monnaie et d'émission }\end{array}$ & $\begin{array}{l}\text { Émission automatique } \\
\text { consubstantielle de l'échange, sous } \\
\text { forme d'un crédit personnel ; } \\
\text { monnaie multilatérale }\end{array}$ & $\begin{array}{l}\text { Émission automatique } \\
\text { consubstantielle de l'échange, sous } \\
\text { forme d'un crédit personnel ; } \\
\text { monnaie multilatérale }\end{array}$ & $\begin{array}{l}\text { Émission a priori et centralisée, par } \\
\text { un crédit à l'adhésion. Mais } \\
\text { possibilité de mettre en œuvre du } \\
\text { crédit pour le développement } \\
\text { d'activités ; monnaie } \\
\text { complémentaire }\end{array}$ & $\begin{array}{l}\text { Émission sous forme de } \\
\text { récompense (par exemple, du } \\
\text { bénévolat ou d'une consommation } \\
\text { responsable), d'aide sociale, de } \\
\text { crédit sur projets, etc.; monnaie } \\
\text { complémentaire }\end{array}$ & $\begin{array}{c}\text { Émission proportionnée aux } \\
\text { demandes de crédit pour } \\
\text { projets d'activité productive (crédit } \\
\text { d'investissement) et aux demandes } \\
\text { de conversion de monnaie nationale } \\
\text { en monnaie locale ; monnaie } \\
\text { complémentaire }\end{array}$ \\
\hline $\begin{array}{l}\text { Mode de garantie de la } \\
\text { monnaie interne }\end{array}$ & $\begin{array}{l}\text { Aucune réserve ; cohésion du } \\
\text { groupe }\end{array}$ & $\begin{array}{l}\text { Aucune réserve ; cohésion du } \\
\text { groupe }\end{array}$ & $\begin{array}{l}\text { Aucune réserve ; cohésion du } \\
\text { groupe }\end{array}$ & $\begin{array}{c}\text { Couverture partielle ou totale de la } \\
\text { monnaie interne par des réserves en } \\
\text { monnaie nationale }\end{array}$ & $\begin{array}{c}\text { Couverture partielle ou totale de la } \\
\text { monnaie locale par des réserves en } \\
\text { monnaie nationale }\end{array}$ \\
\hline $\begin{array}{l}\text { Degré de convertibilité } \\
\text { des avoirs }\end{array}$ & $\begin{array}{l}\text { Inconvertibilité totale des avoirs (ni } \\
\text { entrée ni sortie) }\end{array}$ & $\begin{array}{l}\text { Inconvertibilité totale des avoirs (ni } \\
\text { entrée ni sortie) }\end{array}$ & $\begin{array}{c}\text { Principe d'inconvertibilité des } \\
\text { avoirs parfois atténué avec la } \\
\text { possibilité de transferts vers } \\
\text { l'intérieur }\end{array}$ & $\begin{array}{l}\text { Conversion possible, vers } \\
\text { l'intérieur comme vers l'extérieur, } \\
\text { mais contrôlée par taxe }\end{array}$ & $\begin{array}{l}\text { Conversion possible, vers } \\
\text { l'intérieur comme vers l'extérieur, } \\
\text { mais contrôlée par taxe }\end{array}$ \\
\hline Monnaies utilisables & $\begin{array}{l}\text { Seulement la monnaie locale } \\
\text { (échanges mono-monétaires) }\end{array}$ & $\begin{array}{c}\text { Monnaie locale et monnaie } \\
\text { nationale (échanges bimonétaires) } \\
\text { pour que les professionnels puissent } \\
\text { faire face à leurs frais externes }\end{array}$ & $\begin{array}{l}\text { Seulement la monnaie locale } \\
\text { (échanges mono-monétaires) }\end{array}$ & $\begin{array}{l}\text { Possibilité d'échanges bimonétaires } \\
\text { (monnaie interne et monnaie } \\
\text { nationale) }\end{array}$ & $\begin{array}{c}\text { Monnaie locale et monnaie } \\
\text { nationale (échanges bimonétaires) }\end{array}$ \\
\hline $\begin{array}{l}\text { Degré de substituabilité } \\
\text { externe des biens et } \\
\text { services échangés en } \\
\text { interne }\end{array}$ & $\begin{array}{c}\text { Faible } \\
\text { (Biens et services inaccessibles à } \\
\text { l'extérieur et biens et services } \\
\text { accessibles à l'extérieur mais } \\
\text { faiblement substituables, car dotés } \\
\text { d'une forte charge de relation } \\
\text { personnelle ou accessibles à un coût } \\
\text { jugé prohibitif) }\end{array}$ & $\begin{array}{c}\text { De moyen à fort } \\
\text { (Biens et services accessibles à } \\
\text { l'extérieur, Biens et services } \\
\text { inaccessibles à l'extérieur et biens } \\
\text { et services accessibles à l'extérieur } \\
\text { mais faiblement substituables, car } \\
\text { dotés d'une forte charge de relation } \\
\text { personnelle ou accessibles à un coût } \\
\text { jugé prohibitif) }\end{array}$ & $\begin{array}{c}\text { De moyen à fort } \\
\text { (Biens et services inaccessibles à } \\
\text { l'extérieur et biens et services } \\
\text { accessibles à l'extérieur mais } \\
\text { faiblement substituables, car dotés } \\
\text { d'une forte charge de relation } \\
\text { personnelle ou accessibles à un coût } \\
\text { jugé prohibitif) }\end{array}$ & $\begin{array}{c}\text { Moyen } \\
\text { (Biens et services accessibles en } \\
\text { monnaie nationale, mais alors de } \\
\text { façon plus coûteuse) }\end{array}$ & $\begin{array}{c}\text { Très fort } \\
\text { (Biens et services accessibles à } \\
\text { l'extérieur) }\end{array}$ \\
\hline
\end{tabular}




\section{Bibliographie}

BLANC J., 1998, «Free Money for Social Progress. Theory and Practice of Gesell's Accelerated Money », American Journal of Economics and Sociology, vol. 57, n 4, october 1998, 469-483.

BLANC J., 2000, Les monnaies parallèles. Unité et diversité du fait monétaire, Paris : L'Harmattan.

BLANC J., 2006, dir., Exclusion et liens financiers : Monnaies sociales, rapport 2005-2006, Paris : Economica.

BLANC J., 2007, «Les monnaies sociales : dynamique et logiques des dispositifs », RECMA. Revue internationale de l'économie sociale, $\mathrm{n}^{\circ} 303$, mars 2007, pp. 30-43.

BOWRING F., 2000, «Les SEL et les inégalités sociales », La Revue du Mauss semestrielle, $\mathrm{n}^{\mathrm{o}} 15,1^{\mathrm{er}}$ semestre, pp. 373-382.

DELILlE P., WHITAKER C., 2006, «Le projet Sol : pour retrouver le sens des valeurs », in: J. Blanc, (dir.), Exclusion et liens financiers : Monnaies sociales, rapport 2005-2006, Paris : Economica, pp. 383-394.

DEMEULENAERE S., 2004, «General Factors Which May Influence the Success of a Local Currency System », http://www.complementarycurrency.org/helpdesk/success factors.html

DOUTHWAITE R., 1996, Short Circuit. Strengthening Local Economies for Security in an Unstable World, Foxhole : Greenbooks.

ENNES (European Network of Non-Monetary Exchange Systems), 2001, Without Money. A Guide to Non-Monetary Exchange Systems, Roma : Lunaria.

FERREIRA A., MOERS P., 2006, « Le projet Fomento à Fortaleza », in : J. Blanc, (dir.), Exclusion et liens financiers : Monnaies sociales, rapport 2005-2006, Paris : Economica, pp. 355-382.

FISHER I., 1933, Stamp Scrip, New York : Adelphi.

GESELL S., 1916, L'ordre économique naturel, trad. fr. de la $8^{\mathrm{e}}$ éd. all., Paris : Marcel Rivière, 1948.

GLOVER P., 1995, Hometown Money : How to Enrich Your Community with Local Currency, Ithaca (New York) : Ithaca Money.

GRECO T. H. Jr., 2001, Money: Understanding and Creating Alternatives to Legal Tender, White River Junction (Vermont) : Chelsea Green Publishing.

HART K., 2000, Money in an unequal world. Keith Hart and his memory bank, New York, London : Texere.

HINTZE S., ed., 2003, Trueque y Economía Solidaria, Buenos Aires : UNDP Argentina, UNGS, Prometeo Libros.

KENNEDY M., 1990, Libérer l'argent de l'inflation et des taux d'intérêt. Créer un moyen d'échange que tout le monde puisse utiliser et qui protège la terre, Genève : Éditions Vivez Soleil, 1996. 
KENNEDY M., 2006, «Pourquoi avons-nous besoin d'innovations monétaires? Trois erreurs courantes, trois conséquences probables, trois solutions possibles », in : J. Blanc, (dir.), Exclusion et liens financiers : Monnaies sociales, rapport 2005-2006, Paris : Economica, pp. 99-120.

KEYNES J. M., 1936, The General Theory of Employment, Interest and Money, London : MacMillan.

LAACHER S., 2003, Les SEL. Une utopie anticapitaliste en pratique, Paris : La Dispute.

LEYSHON A., LEE R, WILliAMS C. C., dir., 2003, Alternative Economic Spaces, London, Thousand Oaks, New Delhi : Sage Publications.

LIETAER B., 2001, The Future of Money, London : Random House.

LUZZI M., 2005, Réinventer le marché ? Les clubs de trueque face à la crise en Argentine, Paris : L'Harmattan, coll. : « Logiques sociales ».

MONTILLET L., 2003, «Les monnaies communautaires en Argentine : le trueque. Analyse de l'émergence, la croissance exponentielle et l'essoufflement des mouvements de monnaies communautaires en Argentine », in: I. Guérin, J.-M. Servet, (dir.), Rapport Exclusion et liens financiers 2003-2004, Paris : Economica, pp. 137-158.

MONTILlET L., 2006, «Et pour quelques créditos de plus... », in: J. Blanc, (dir.), Exclusion et liens financiers : Monnaies sociales, rapport 2005-2006, Paris : Economica, pp. 401-422.

ONKEN W., 1997, Modellversuche mit sozialpflichtigem Bodem und Geld, Lütjenburg: Fachverlag für Sozialökonomie.

PLASENCIA A., ORZI R., eds, 2007, Moneda social y mercados solidarios. Potencial emancipador y pedagógico de los sistemas monetarios alternativos, Buenos Aires : Ediciones Ciccus.

RIZZO P., 2000, L'imaginaire multilatéral. Système d'interinformation et reliance multilatérale. Profils sémantiques de LETS, SEL, TR, BdT, SRI, Thèse de doctorat en sociologie, Grenoble : Université Pierre Mendès France, Grenoble 2 (CSRPC).

SERVET J.-M., 1999, dir., Une économie sans argent. Les systèmes d'échange local, Paris : Seuil.

THÉRET B., ZANABRIA M., 2007, « ¿Es posible un federalismo monetario viable? », in : R. Boyer, J. Neffa, (dir.), Salidas de crisis y estrategias de desarrollo viables para Argentina, Buenos Aires : Miño y Davila.

TOFFLER A, 1980, La troisième vague, Paris : Denoël / Gonthier.

VAN ARKEL H., RAMADA C., VINK J., 2006, « Méthodes pour le succès des monnaies complémentaires », in : J. Blanc, (dir.), Exclusion et liens financiers : Monnaies sociales, rapport 2005-2006, Paris : Economica, pp. 213228. 
WILLIAMS C. C., ALDRIDGE T., LEE R., LEYSHON A., THRIFT N. ET TOOKE J., 2001, Bridges into Work? An Evaluation of Local Exchange and Trading Schemes (LETS), Bristol : Policy Press. 